\title{
PENSER ET ÊTRE: \\ LA CRITIQUE SARTRIENNE DU COGITO*
}

Philippe Cabestan

Lycée Lavoisier-Paris

Sans doute est-il artificiel de vouloir retrouver dans les premiers textes d'un philosophe l'ensemble de sa pensée. Comme si le temps écoulé et les événements qui l'ont ponctué ne comptaient pour rien dans l'élaboration de l'œuvre. Et pourtant, il est rare qu'un philosophe dénonce ses premières œuvres - qu'il persiste, au 'contraire, à signer au prix parfois d'une auto-interprétation discutable.' Sartre n'échappe pas à la règle. En dépit de quelques déclarations rapportées par les uns ou les autres, la palinodie n'est à vrai dire pas son genre, et nous sommes tentés de penser non seulement que Sartre n'a jamais réellement renié ses premières recherches phénoménologiques mais, en outre, que celles-ci lui offrent les assises de sa pensée, son sol véritable.

En effet, si on peut non sans raison distinguer un premier et un deuxième Sartre voire un troisième Sartre, reste que la Critique de la raison dialectique aussi bien que les essais consacrés à Baudelaire, Jean Genet, Mallarmé ou Flaubert - sans parler du théâtre et des textes littéraires comme Le Mur ou La nausée - reprennent inchangées les thèses phénoménologiques auxquelles Sartre pour l'essentiel ne renonce jamais et qu'il expose notamment dans L'être et le néant. Par exemple, c'est dans la quatrième et dernière partie de L'être et le néant que Sartre expose son projet

* Ce texte correspond à la version remaniée d'une présentation de la Phénoménologie sartrienne dans le cadre d'un cycle de Séminaires organisé par le Projet de Recherche «Phénoménologie et Ontologie " (coordonné par le Professeur Isabel Matos Dias) du Centro de Filosofia da Universidade de Lisboa, à la Faculté de Lettres de l'Université de Lisbonne, les 30 et 31 Mars 2000.

' C'est toute la question de la Kehre dans l'œuvre de Heidegger. Jean Grondin, Le tournant dans la pensée de Martin Heidegger, Paris, Puf, 1987. 
de psychanalyse existentielle qui trouve à son tour ses principes dans les recherches ontophénoménologiques exposées au cours des trois premières parties. De même, on pourrait montrer sans grande difficulté de quelle manière la Critique de la raison dialectique s'articule à L'être et le néant, et tente de s'élever au plan collectif et historique en partant de l'individu en tant que pour soi et libre práxis.

Aussi voudrions-nous dans cette brève présentation de la pensée sartrienne, plutôt que de survoler l'ensemble de l'œuvre, nous limiter à son point de départ et tenter d'élucider de quelle manière la phénoménologie permet à Sartre d'élaborer un nouveau cogito en redéfinissant le rapport de la conscience à l'être, mieux: au néant. Dans un premier temps, nous étudierons la notion de réflexion et le projet sartrien d'une purification de la conscience. Puis, suivant le fil conducteur de la notion de mauvaise foi, nous tenterons de dégager le mode d'être de la réalité-humaine. Enfin, l'analyse du cogito préréflexif nous permettra de dévoiler le néant qui habite la conscience sartrienne en tant que présence à soi.

\section{La notion de réflexion}

Sartre a découvert la phénoménologie de manière relativement tardive, grâce à Levinas, quoique ce dernier fût légèrement plus jeune que lui, et à son livre: La théorie de l'intuition dans la phénoménologie de Husserl (1930). ${ }^{2}$ Il n'est peut-être pas ici nécessaire d'évoquer la fameuse soirée au Bec de Gaz et son cocktail à l'abricot. ${ }^{3}$ On se contentera de rappeler que Sartre succède à Raymond Aron à l'institut français de Berlin en 1933, année au cours de laquelle il se plonge dans la lecture de Husserl et, notamment, des Recherches logiques et des Idées directrices pour une phénoménologie. Ce fut sans aucun doute une rencontre déterminante qui le conduisit un an après à rédiger son célèbre article repris dans Situations I: «Une idée fondamentale de la phénoménologie de Husserl: l'intentionnalité » et La transcendance de l'ego (1934). ${ }^{4}$ C'est de même avec et contre Husserl qu'il écrit L'imagination (1936), l'Esquisse d'une théorie des émotions (1939) et L'imaginaire (1940). Dans ses premiers textes et, en particulier, dans La transcendance de l'ego, Sartre expose déjà sa conception, décisive pour ses recherches futures, de la réflexion pure. En effet, comme

2 E. Levinas, La théorie de l'intuition dans la phénoménologie de Husserl, Paris, Vrin, 1989.

S. de Beauvoir, La force de l'âge, Paris, Gallimard, 1960, pp.156-7.

4 J.-P. Sartre, Situations I, «Une idée fondamentale de la phénoménologie de Husserl: l'intentionnalité », Paris, Gallimard, 1975; La transcendance de l'ego, Paris, Vrin, 1988. Pour une étude approfondie de ces textes, nous renvoyons à V. de Correbyter, Sartre face à la phénoménologie, Bruxelles, Ousia, 2000. 
nous allons le voir, seule la réflexion pure rend possible une authentique phénoménologie.

\section{a. Le préréflexif}

Husserl ne cesse tout au long de sa vie de revenir sur les présupposés méthodologiques de la phénoménologie transcendantale et de s'interroger sur la nature de la réduction phénoménologique. Ainsi, à la fin de sa vie, dans La crise des sciences européennes et la phénoménologie transcendantale (1936), Husserl s'interroge encore et toujours sur les voies de la réduction parmi lesquelles il distingue la voie cartésienne, la voie du monde de la vie et la voie de la psychologie. À l'opposé, les textes méthodologiques de Sartre concernant les conditions d'une description phénoménologique rigoureuse - nous laissons de côté par conséquent Questions de méthode ${ }^{5}$ - sont relativement rares. Tout se passe comme si, aux yeux de Sartre, Husserl s'était suffisamment attardé sur ces questions méthodologiques et qu'il importait à présent d'aller de l'avant. Cependant, il n'est pas vrai que Sartre se contente de reprendre telle quelle la conception husserlienne de la réduction. En effet, dans le prolongement des recherches husserliennes sur la réflexion naturelle et la réflexion transcendantale, Sartre distingue entre deux types de réflexion: la réflexion pure et la réflexion impure, distinction qui repose elle-même sur une première distinction entre l'attitude préréflexive et l'attitude réflexive, le cogito préréflexif et le cogito réflexif.

Quand, pressée, je regarde l'heure ou que je ramasse un objet qui vient de tomber, ou encore lorsque je cours après un tramway, ma conscience est essentiellement conscience de l'objet intentionnel qu'elle vise, par exemple, du «-devant-être-rejoint». ${ }^{6}$ En d'autres termes, ce qui caractérise l'attitude préréflexive c'est que je suis comme plongé dans le monde des objets, comme capté par le, la montre, l'objet-tombé-à-ramasser, etc. L'abandon de l'attitude irréfléchie au profit de la réflexion implique alors un changement fondamental de la structure de la conscience. Dans l'attitude réflexive, la conscience fait retour sur elle-même, elle se prend désormais pour objet intentionnel. Dès lors, j'ai conscience non plus du tramway mais de vouloir rattraper le tramway, mieux: la conscience réflexive est conscience du vécu (Erlebnis) qui est le mien lorsque je cours après le tramway. De même, au cours de la lecture d'un roman, ma conscience est captée par le héros et ses différentes aventures, mais je puis toujours, à moins d'être totalement pris par le roman, prendre conscience que je lis et ma conscience est alors conscience de la conscience de lecture. 
On peut décrire l'attitude préréflexive de manière plus rigoureuse en disant d'une part que, conformément au principe de l'intentionnalité selon lequel toute conscience est conscience de quelque chose, la conscience préréflexive est conscience positionnelle d'un objet transcendant; et, d'autre part, que la conscience est nécessairement conscience non positionnelle d'elle-même, de son vécu immanent. C'est pourquoi, si on me demande ce que fais, tandis que je m'abandonne par exemple à la rêverie, il m'est toujours possible d'indiquer la nature de mon activité. Car ma conscience en tant que conscience irréfléchie de «rêver» est à la fois conscience positionnelle - ou plus précisément quasi positionnelle-de l'objet de ma rêverie, et conscience non positionnelle de l'acte de rêver. Que la conscience positionnelle soit inséparable d'une conscience non positionnelle de soi, c'est ce que manifeste bien le fait que je sais immédiatement si ce que je vois je le perçois ou l'imagine: à chaque fois, présent à moi-même, j'ai une conscience non positionnelle, Sartre dit également « non thétique, de mes actes intentionnels.

La réflexion signifie alors que la conscience devient conscience positionnelle d'elle-même, c'est-à-dire qu'elle se dédouble, tout en conservant son unité fondamentale, en une conscience réfléchissante et une conscience réfléchie qui devient objet de la conscience réfléchissante. Ainsi, dans la réflexion, la conscience réfléchissante est conscience positionnelle de la conscience réfléchie et, en même temps, conscience non positionnelle d'elle-même. ${ }^{7}$ Il va de soi que nous pouvons également envisager avec Sartre la possibilité d'une réflexion au deuxième degré, autrement dit une réflexion de la réflexion qui permet à la conscience réfléchissante de devenir l'objet réfléchi d'une nouvelle conscience réfléchissante qui est en même temps conscience non positionnelle d'elle-même. Pour marquer nettement la différence entre le rapport de la conscience à l'objet positionnel qu'elle vise et son rapport non positionnel à soi, L'être et le néant oppose la conscience de quelque chose et la conscience (de) soi, en mettant dans ce dernier cas le (de) entre parenthèses.

\section{b. Réflexion et subjectivité pures}

Nous pouvons à présent distinguer avec Sartre entre deux types de réflexion: la réflexion impure ou complice et la réflexion pure ou non complice, distinction dont on ne saurait trop souligner l'importance méthodologique. Sartre affirme en effet: "Toute notre ontologie a son fondement dans une expérience réflexive $\gg .^{8}$ Cependant, il importe de bien saisir en l'occurrence de quelle expérience il s'agit. 
En faisant retour sur elle-même, la conscience réfléchissante peut être tentée de constituer la conscience réfléchie en un objet et, ce faisant, de dénaturer la subjectivité au point d'en faire une réalité mondaine - ce que Husserl appelle: «ein weltliches Real »-voire substantielle. C'est précisément ce que font les psychologues qui étudient la psychè ou le psychisme en tant qu'objet et qui se placent du point de vue de la réflexion impure. ${ }^{9}$ Pour la psychologie, en effet, la psychè est un quelque chose auquel est attribué des états tels que l'amour et la haine, des qualités comme le fait que le sujet est rancunier, jaloux, généreux, et des actions telles que jouer au piano, s'entraîner à la boxe, enseigner. Remarquons qu'il s'agit à chaque fois d'objectités transcendantes et constituées, qui débordent largement le champ actuel de la conscience et qui sont en tant que telles hors de la conscience, transcendantes. Pour bien comprendre ce point, nous pouvons distinguer, par exemple, entre la répulsion que j'éprouve en voyant Pierre en tant que vécu de conscience immanent et la haine que j'éprouve pour Pierre qui est un objet transcendant constitué à partir des vécus de répulsions par la réflexion impure.

Mais si le psychisme des psychologues est chose parmi des choses à l'instar de la table ou de la chaise, un être-au-milieu-du-monde dont l'apparaître est relatif, et qui est actif ou passif selon qu'il agit ou subit, la conscience est, du point de vue de la réflexion pure, une pure spontanéité, un absolu non substantiel. En effet, dans la réflexion pure, la conscience réfléchissante s'en tient strictement au vécu intentionnel, à ce qui apparaît, sans se permettre d'aller subrepticement au-delà. Ainsi la réflexion pure résulte d'une sorte de catharsis ou purification de la réflexion qui est généralement impure. Corrélativement, Sartre distingue deux sphères d'évidence. D'une part, et nous retrouvons naturellement une distinction établie par Husserl dès Les Recherches logiques, une sphère d'évidence adéquate qui correspond au domaine de la réflexion pure: dans ce cas en effet l'intuition du vécu intentionnel offre un remplissement (Erfühlung) parfait de l'intention signifiante. D'autre part, une sphère d'évidence inadéquate dont l'inadéquation est identique à celle qui caractérise la perception de n'importe quel objet transcendant qui se livre par profils ou Abschattungen. C'est pourquoi, de même que la perception d'une table est et restera toujours douteuse, de même la haine ou l'amour sont des sentiments certes réels (real) mais douteux qui se donnent comme objets transcendants à travers une série jamais achevée de vécus: rien ne me garantit que demain ma haine pour Pierre ne se soit pas évanouie.

Après avoir distingué entre conscience préréflexive et conscience réflexive, réflexion pure et réflexion impure, La transcendance de l'ego peut énoncer la thèse que L'être et le néant approfondit d'un point de vue on- 
tologique: la subjectivité véritable est pure de tout ego psychique, de tout état ou qualité en sorte que plus rien ne saurait troubler sa transparence comme sa vacuité. Remarquons à ce propos que, peut-être en raison du titre de l'ouvrage, on croit souvent que La transcendance de l'ego n'a d'autre objet que d'exclure l'ego de la sphère d'immanence et de lui reconnaître sa transcendance. On oublie alors ce que, dans la conclusion même de ce texte, Sartre indique nettement la fin qu'il poursuit à travers cette exclusion: « La conception de l'Ego que nous proposons nous paraît réaliser la libération du champ transcendantal en même temps que sa purification. Le champ transcendantal, purifié de toute structure égologique, recouvre sa limpidité première. En un sens c'est un rien puisque tous les objets physiques, psycho-physiques et psychiques, toutes les vérités, toutes les valeurs sont hors de lui, puisque mon Moi a cessé, lui-même, d'en faire partie. Mais ce rien est tout puisqu'il est conscience de tous ces objets». ${ }^{10}$

De manière analogue, dans son article publié en 1939 mais qui fut vraisemblablement rédigé en 1934, Sartre expose avec enthousiasme de quelle manière l'intentionnalité husserlienne permet de purifier la conscience. Il y écrit notamment: « Du même coup, la conscience s'est purifiée, elle est claire comme un grand vent, il n'y a plus rien en elle, sauf un mouvement pour se fuir, un glissement hors de soi; si, par impossible, vous entriez 'dans' une conscience, vous seriez saisi par un tourbillon et rejeté au-dehors, près de l'arbre, en pleine poussière, car la conscience n'a pas de 'dedans'; elle n'est rien que le dehors d'elle-même et c'est cette fuite absolue, ce refus d'être substance qui la constituent comme une conscience ${ }^{11}$. Nous devinons déjà comment $L$ 'être et le néant peut assimiler la conscience au néant, mieux: à un acte néantisant.

\section{La mauvaise foi}

Conduite sans prendre en compte la dimension corporelle de la conscience et, via le corps, son inscription factuelle dans le monde, cette description de la conscience reste fondamentalement abstraite. En outre, son mode d'être nous demeure obscur, et si nous savons déjà qu'elle est une pure spontanéité, un absolu non substantiel, reste à élucider la signification de concepts aussi problématiques du point de vue de l'ontologie naturaliste qui régit la science moderne de la nature. Aussi voudrions-nous à présent envisager de quelle manière L'être et le néant approfondit cette description de la conscience en vue d'un nouveau cogito. Essai d'ontologie phénoménologique, L'être et le néant se propose en effet d'élucider ces deux régions d'être, mises en évidence dès son introduction, que sont le phéno-

${ }_{11}^{10}$ La transcendance de l'ego, p.74.

${ }^{11}$ Situations I, p.40. 
mène et ce à quoi apparaît le phénomène, la chose et la conscience, l'en-soi et le pour-soi. ${ }^{12}$ Mais parce que, comme nous l'apprend Heidegger, les êtres ne partagent pas nécessairement le même mode d'être, l'élucidation du sens d'être du phénomène ne saurait avoir d'emblée une quelconque validité pour ce qui concerne l'être de la conscience. Pour Sartre, "L'être de la conscience étant radicalement autre, son sens nécessitera une élucidation particulière à partir de la révélation révélée d'un autre type d'être, l'être pour soi, que nous définirons plus loin $» .^{13} \mathrm{Il}$ faut donc reconnaître entre l'en-soi et le pour-soi une différence ontologique.

Afin d'élucider le sens d'être de phénomènes comme cette pierre, ce marronnier, cette table ou cette chaise, il nous faut partir de « la claire vision du phénomène d'être », en prenant donc appui sur l'être du phénomène tel qu'il se manifeste. Pour Sartre, cette manifestation ne souffre pas d'ambiguïté: "l'être est en soi ». Cette formule n'en possède pas moins plusieurs significations. Dire de l'être du phénomène qu'il est en soi signifie tout d'abord que l'être est incréé; il est son propre support (selbstständig), non pas au sens où il serait cause de soi, ce qui y introduirait déjà un rapport à soi, mais au sens où il est soi. Et étant soi, l'être est précisément sans rapport à soi. De ce point de vue, il ne peut être ni affirmation de soi ni négation de soi. L'être est en soi ou encore "l'être est ce qu'il est », expression qui n'énonce pas le simple principe d'identité de la logique formelle mais qui constitue un principe régional qui, nous allons le voir, ne s'applique pas à cette autre région de l'être qu'est le pour-soi. Enfin, comme le révèle la nausée, l'être est contingent, c'est-à-dire qu'on ne saurait le dériver de quoi que ce soit qui lui fournirait sa raison d'être. Il est donc sans raison d'être, sans nécessité. Exprimé en termes anthropologiques, l'être est de trop, superflu. Cette description du phénomène d'être s'étend dans L'être et le néant sur un peu plus de cinq pages. Par la suite Sartre n'en dira guère plus et l'explicitation du sens d'être du phénomène semble donc achevée.

En revanche, la description des structures du pour-soi telles qu'elles se donnent dans le cogito occupe quelque 250 pages - et nous ne prenons même pas en compte l'être-pour-autrui du pour-soi. Il va donc de soi que nous ne pouvons procéder ici à un exposé exhaustif, et nous nous contenterons de dégager, d'un point de vue ontologique, la structure première de la présence à soi. Dans cette perspective, il nous faut analyser le phénomène de la mauvaise foi qui occupe dans l'économie de L'être et le néant une place fondamentale - plus fondamentale même que l'angoisse - au point que Sartre a pu par la suite présenter L'être et le néant comme une eidéti-

${ }^{12}$ Ainsi que la région de l'en-soi-pour-soi qui correspond à la sphère idéale de l'ens causa sui en tant que projet du pour-soi.

${ }^{13}$ L'être et le néant, p.30. 
que de la mauvaise foi $^{14}$. Comme nous allons le voir, alors que l'intuition de l'être du phénomène nous révèle un être qui est ce qu'il est, un être en soi, l'analyse de la mauvaise foi nous permet d'entr'apercevoir le sens d'être de la réalité-humaine que le cogito permettra de fixer dans une évidence irrécusable. Car, pour le dire par avance, « la condition de possibilité de la mauvaise foi, c'est que la réalité humaine, dans son être le plus immédiat, dans l'infrastructure du cogito préréflexif, soit ce qu'elle n'est pas et ne soit pas ce qu'elle est $\rangle^{15}$. Il nous faut donc essayer de comprendre, en prenant l'analyse de la mauvaise foi pour fil conducteur, que la conscience est cet être qui n'est pas ce qu'il est tout en étant ce qu'il n'est pas.

Si le mensonge suppose la dualité du trompeur et du trompé, la mauvaise foi est mensonge à soi. Être de mauvaise foi, c'est donc se mentir à soi-même. Parmi les différents exemples de conduites de mauvaise foi exposés dans L'être et le néant, nous pouvons privilégier celui de la femme frigide que Sartre emprunte à l'un des premiers disciples de Freud, W. Steckel. Les recherches de ce dernier sur la frigidité l'intéressent d'autant plus que, psychanalyste dissident, Steckel renonce finalement à l'hypothèse freudienne de l'inconscient. Ainsi Sartre peut lire dans La femme frigide (1937): « Ne croyez pas les malades. Soyez méfiants, sceptiques parce que vos clients sont de très bons acteurs, jouant la comédie devant le médecin, mais la jouant aussi vis-à-vis d'eux-mêmes ». ${ }^{16}$ En d'autres termes, la frigidité de certaines femmes relève non de la pure et simple comédie, qui suppose comme le mensonge la claire conscience de ce qu'on fait, mais d'une mauvaise foi pathologique qui permet à la femme de se masquer la jouissance que lui procure l'acte sexuel. Par exemple, la femme fait les comptes du ménage pour ne pas ressentir de plaisir et se prouver qu'elle est frigide.

Afin d'appréhender plus précisément le phénomène de la mauvaise fois, il nous faut repartir de la structure duale de l'existence qui est inséparablement transcendance et facticité. Transcendance, au sens où l'existence est ce mouvement constant par lequel le sujet échappe à ce qu'il est en se faisant autre que ce qu'il est, en n'étant pas ce qu'il est tout en étant ce qu'il n'est pas. Facticité, car cette existence en tant que surgissement à l'être possède une dimension de fait qui échappe à la liberté: elle est. Si je puis toujours choisir de mettre fin à mes jours et de ne plus être, nul n'a en revanche choisi de naître en tel lieu à telle époque dans telle famille ou telle communauté. ${ }^{17}$ La facticité désigne ainsi le fait que je suis, le fait de mon existence, à partir duquel une conduite est possible. Appliqués à la mau-

${ }_{11}^{14}$ J.-P. Sartre, Situations IV, Paris, Gallimard, 1964, p.196.

${ }_{16}^{15}$ L'être et le néant, p.104

${ }^{16}$ W. Steckel, Die Geschlechtskälte der Frau, trad. J. Dalsace, La femme frigide, Paris, Gallimard, 1975.

${ }^{17}$ Il faudrait en toute rigueur distinguer les concepts de facticité et de situation, mais cela ne nous semble pas ici nécessaire. 
vaise foi, ces deux concepts permettent de la définir comme un jeu « sournois » qui consiste à « affirmer la facticité comme étant la transcendance et la transcendance comme étant la facticité, de façon qu'on puisse, dans l'instant où on saisit l'une, se trouver brusquement en face de l'autre». ${ }^{18}$ Ainsi, la femme frigide fait comme si sa frigidité était une maladie, un état relevant de sa facticité alors qu'elle est une conduite, issue du projet de ne pas éprouver de plaisir, qui relève de sa transcendance, c'est-à-dire précisément de son pouvoir de n'être pas ce qu'elle est. La femme frigide se ment donc à elle-même en se disant qu'elle est frigide et en niant sa libre transcendance.

Mais ce qui nous importe ici avant tout, c'est de comprendre comment la mauvaise foi est possible; comment une femme peut se mentir à elle-même au point de dire qu'elle est frigide, alors même qu'elle éprouve du plaisir, et de consulter dans certains cas un médecin. Sartre nous répond: « Si la mauvaise foi est possible, à titre de simple projet, c'est que, justement, il n'y a pas de différence si tranchée entre être et n'être pas, lorsqu'il s'agit de mon être ». En d'autres termes, si la femme frigide peut former le projet d'être frigide alors même qu'elle éprouve du plaisir, c'est fondamentalement parce le plaisir qu'elle fuit n'a pas la consistance d'une chose en soi, la densité et l'opacité d'un être qui est ce qu'il est. C'est parce que ce plaisir, comme tout plaisir et toute douleur humaine, est lui-même en question dans son être, qu'il est possible de former un projet de mauvaise foi. Si par impossible le plaisir pouvait avoir le mode d'être massif et sans faille ou distance à soi de la chose, un tel projet serait inenvisageable. Ainsi la mauvaise foi est possible parce que le mode d'être de la conscience qui éprouve du plaisir n'est pas celui de la chose en soi, et la mauvaise foi exige à titre de condition ontologique de possibilité, « que je ne sois pas ce que je suis, c'est-à-dire qu'il y ait une différence impondérable qui sépare l'être du non-être dans le mode d'être de la réalité-humaine». ${ }^{19}$ Ainsi les conduites de mauvaise foi reposent sur une précompréhension de la spécificité du mode d'être de la conscience qui, à la différence de la chose en soi qui est ce qu'elle est, n'est pas ce qu'elle est et est ce qu'elle n'est pas. Et la mauvaise foi consiste alors à refuser le mode d'être qui est le sien.

C'est également ce que révèle la manière dont certains se rapportent à leur propre passé ou à leur propre situation. En effet, alors que la réalité-humaine est son passé sur le mode de l'être qui n'est pas ce qu'il est et qui est ce qu'il n'est pas, certains sont néanmoins tentés de refuser leur propre passé au point d'affirmer qu'ils ne sont pas (ou plus) ce qu'ils étaient à la manière dont la chaise n'est pas la table. Par exemple, face à un reproche, je soutiens avoir changé et ne plus être celui qui a commis l'acte en question. Mais, inversement, un homme de mauvaise foi peut également pré- 
tendre être son passé sur le mode de l'en-soi qui est ce qu'il est. Dans ce cas - comme ces combattants de la première guerre mondiale qui le sont restés jusqu'à la fin de leurs jours, - il affirme être ce qu'il était au point de nier le néant temporel qui le sépare de ce qu'il était. Il en va de manière analogue dans le rapport que certains entretiennent avec leur profession, les uns s'efforçant de coïncider avec leur être social tandis que d'autres le fuient. Ainsi le garçon de café joue à être garçon de café et, ce faisant, nie sa transcendance au profit de sa facticité. Il voudrait être garçon de café en soi et non pour soi. Nous retrouvons dans ces différents cas la structure de la mauvaise foi: refuser d'être ce qu'on est sur le mode spécifique qui est celui de la conscience dont chaque sujet possède une précompréhension. C'est à partir de cette précompréhension qu'il est possible de dégager la structure ontologique de la conscience.

\section{Un cogito purifié}

Dans le prolongement des philosophies de la conscience et en opposition à l'analytique existentiale de Heidegger, L'être et le néant affirme la nécessité pour toute philosophie rigoureuse de prendre son point de départ dans le cogito. Mais, comme nous voudrions à présent le montrer, le cogito sartrien n'est ni celui de Descartes ni celui de Husserl.

Sartre demeure en un sens fidèle à Husserl auquel il emprunte un ensemble d'oppositions: celles de l'immanent et du transcendant, de l'évidence adéquate et de l'évidence inadéquate, du réflexif et du préréflexif. Sartre n'a pas non plus oublié que Husserl - après Kant et à la différence de Descartes - distingue le transcendantal et le psychologique et, par suite, un cogito transcendantal, fruit de la réduction transcendantale, et un cogito psychologique qui relève de l'attitude naturelle. Mais apparaît déjà une première différence: pour Husserl, la conscience psychologique résulte non de la réflexion impure mais d'une aperception d'un type original qui, dans la constitution des êtres animés (Animalien), réalise (realisieren) la subjectivité en la liant au corps. ${ }^{20}$ Ainsi, insérée dans le monde réel (real), la conscience perd son immanence et devient une réalité transcendante, une âme (Seele) unie à un corps. Pour Sartre, comme nous l'avons vu, si la psychè est bien un objet transcendant appartenant au monde, sa constitution est indépendante de son incarnation et relève de cette réflexion constituante que Sartre oppose à la réflexion purifiante. Cette dernière est au principe du cogito sartrien.

C'est en effet à partir de l'idée d'une catharsis de la réflexion et d'un cogito qui n'exige pas tant la mise entre parenthèses du monde qu'une 
purification radicale de tout ce qui est étranger à la conscience que Sartre se démarque de Husserl. De ce point de vue, Sartre rejette toute conception égologique de la conscience, et s'oppose aussi bien à Husserl qu'à Kant ou Descartes. $^{21}$ Les uns comme les autres confondent, tout d'abord, la structure du cogito préréflexif et celle du cogito réflexif. En d'autres termes, lorsque dans ses Méditations métaphysiques Descartes écrit: « je pense, je suis », il se place manifestement d'un point de vue réflexif, et son affirmation décrit non la conscience ou la pensée en général mais la conscience réfléchie telle qu'elle apparaît sous le regard de la conscience réfléchissante. Et il en va de même lorsque Kant déclare dans la Critique de la raison pure que le «je pense » doit pouvoir accompagner toutes mes représentations. En outre, faute de distinguer rigoureusement entre la réflexion pure et la réflexion impure, Descartes, Kant et Husserl accordent à celle-ci les droits inaliénables de celle-là. Il n'est pas étonnant dans ces conditions qu'ils définissent la conscience comme une substance et qu'ils réintroduisent au sein du cogito un ego qui, du point de vue de la réflexion pure, n'y a pas sa place. En effet qu'est-ce que l'ego sinon une unité transcendante, constituée par la réflexion impure, qui échappe en tant que telle à toute évidence adéquate et qui s'évanouit sous le regard de la réflexion pure ?

Enfin, la description du cogito préréflexif dans les limites de la réflexion pure permet d'en exclure non seulement tout objet transcendant mais aussi toute forme de passivité en en dévoilant l'absolue spontanéité. Et nous sommes désormais en mesure d'éclaircir la signification de ce dernier concept. Comme nous permet de le comprendre l'explicitation des conduites de mauvaise foi, dont elle constitue la condition de possibilité, et comme nous le dévoile dans une évidence adéquate l'analyse du cogito préreflexif, l'impossibilité de coöncider avec soi, c'est-à-dire d'être ce qu'on est, constitue le mode d'être même de la conscience. En effet, si toute conscience est conscience de quelque chose, elle est en même temps et nécessairement conscience (de) soi. Autrement dit toute conscience est conscience de quelque chose tout en étant « présence à soi ». La présence à soi implique donc une certaine dualité et comme un « décollement de l'être par rapport à soi ». La question est alors de savoir ce qui peut bien séparer le sujet de lui-même et constituer cette distance sur fond d'unité que manifeste la présence à soi. Cela ne saurait être un quelque chose qui serait de l'ordre de l'en-soi. Il ne peut s'agir, à vrai dire, que d'un rien, d'un négatif pur, c'est-à-dire d'un néant d'être. Ainsi, la description ontophénoménologique du cogito préréflexif nous révèle que le mode d'être de la conscience résulte d'un acte néantisant premier ou, si l'on préfère, que la conscience surgit à l'être par une sorte de décompression d'être. La spontanéité de la

${ }^{21}$ On sait que les Idées directrices pour une phénoménologie, contrairement à la première édition des Recherches logiques, réintroduisent l'ego au cœur de la subjectivité transcendantale, en tant que transcendance dans l'immanence. 
conscience désigne rien de moins que cette néantisation première "par quoi l'en-soi se dégrade en présence à soi». ${ }^{22}$

\section{Conclusion}

Tout en s'inscrivant dans le prolongement la pensée de Descartes, Kant et Husserl, la phénoménologie sartrienne trouve son point de départ dans un nouveau cogito à la fois purifié et désubstantialisé. Désormais il n'est plus possible de confondre la conscience et sa réification sous la forme de la psychè. Mais reconnaissons qu'une telle purification et désubstantialisation de la conscience ne vont pas sans susciter quelques difficultés. Affirmer qu'il n'y a rien dans la conscience pour la simple raison qu'elle n'a pas de dedans, et que la conscience n'est rien que le dehors d'elle-même, implique que 1'on renonce à toute idée de passivité, qui suppose l'action d'une chose sur une autre. Car comment une chose agirait-elle sur ce qui n'est rien? Dans ce contexte ontologique, on peut se demander comment il est encore possible de rendre compte des phénomènes dans lesquels la subjectivité semble éminemment passive et que Descartes dénomme pour cette raison des passions de l'âme. En d'autres termes, comment concilier le mode d'être de la conscience dont le néant exclut toute passivité, et des phénomènes tels que l'émotion, la jalousie, le désir ou encore le rêve $?^{23}$ Et nous n'évoquons même pas ces phénomènes pathologiques comme la phobie, l'obsession ou le délire psychotique. Ne doit pas alors reconnaître à la réflexion certaines limites et admettre avec $\mathrm{P}$. Ricœur que le cogito est intérieurement brisé? ${ }^{24}$

${ }^{22}$ L'être et le néant, p.117.

${ }^{23}$ Précisons que c'est à ces difficultés que tente de répondre Sartre dans l'Esquisse d'une théorie des émotions et dans L'imaginaire.

${ }^{24}$ P. Ricœur, Soi-même comme un autre, Paris, Seuil, 1990, p.22. 